\title{
Diagnostic utility of endoscopic brush cytology in upper gastrointestinal lesions and its correlation with biopsy.
}

\author{
${ }^{1}$ Dr Pragati Karmarkar, ${ }^{2}$ Dr Anne Wilkinson, ${ }^{3}$ Dr Tanuja Manohar, \\ ${ }^{4}$ Dr Archana Joshi, ${ }^{5}$ Dr Sadhana Mahore \\ ${ }^{1,2,4}$ Associate Professor Pathology, ${ }^{3}$ Associate Professor Medicine, ${ }^{5}$ Professor and Head, Dept of Pathology, \\ NKP Salve Institute of Medical Sciences, Hingna, Nagpur 440019, Maharashtra, India
}

\begin{abstract}
Background: The endoscopic examination of upper gastrointestinal lesions is invaluable in the diagnosis of different neoplastic and non neoplastic conditions. Malignancies of upper GIT are one of the leading causes of death worldwide. Early cancer is asymptomatic and highly curable. The advent of the endoscope has greatly facilitated the detection and diagnosis of upper GIT lesions. Brush cytology and biopsy are complementary, and yield is higher when both are employed rather than when one is used.
\end{abstract}

Aim: The aim of this study was to analyze the advantages, utility and limitations of brush cytology in upper GIT lesions.

Materials and Methods: 100 cases including neoplastic and non neoplastic lesions, were studied over a period of one year. Endoscopy was done by using flexible video endoscope. Patients with visible mucosal lesions such as ulcers, polypoidal or ulcerative growths in the upper GIT were included. An endoscopic brush followed by biopsy of the lesion was done. The cytological and histopathological findings were compared and the percentage positivity of brush cytology was determined.

Results: Patients between 20-80 years age presented with complaints of dysphagia, retrosternal pain, vomiting and loss of weight. The overall adequacy of brush cytology was 100\%. Smears were interpreted as inflammatory, fungal infection, dysplastic lesions, suspicious for malignancy and positive for malignancy. Biopsy was available in 67 cases of which cytohistological correlation was there in 61 [ $83.68 \%$ ] cases, as the remaining biopsies were inadequate for reporting. .

Conclusion: Endoscopic brush cytology is an effective method for evaluation and screening of upper GIT lesions and can be utilized for rapid diagnosis with minimal discomfort to the patient.

Key words: Brush cytology, upper GIT lesions, Endoscopy

\section{Introduction}

Upper GIT is a common site for various lesions, especially malignant tumours. In India, according to the National Cancer Registry, esophageal and gastric cancers are the most common cancers found in men, while esophageal cancer ranks third among women after the carcinoma of breast and cervix. ${ }^{[1,2,3]}$ In recent years evaluation of endoscopically obtained brush cytology samples has placed the cytopathologist at the forefront of the patient management team in decisions about the treatment of GIT lesions. ${ }^{[4]}$ The diagnostic value of upper GIT biopsy is well established but the value of brush cytology is still a subject of controversy. ${ }^{[4,5]}$ Brush cytology often complements and increases the sensitivity and specificity of detection of GIT lesions in many ways such as endoscopic - guided balloon abrasive technique, lavage, or FNA performed under either endoscopic guidance or computed tomographic guidance. However brush cytology is the most frequently used means of obtaining a sample for the diagnosis of GIT lesions. ${ }^{[4,5]}$ Brushing of the mucosa covers a larger surface area, provides rapid interpretation and early diagnosis. It is less invasive and a risk-free technique compared to biopsy. It is more useful when the lesion is large or multiple or when patients refuse biopsy. It has also the advantage of penetrating to the basement membrane and collecting cells from all the epithelial cell layers of mucosa. ${ }^{[1,6]}$ The present study was done to analyze the advantages as well as the difficulties of brush cytology and to utilize it as a reliable screening tool for rapid diagnosis of upper GIT lesions. ${ }^{[6]}$

\section{Materials And Methods}

The present study was conducted in the Department of Pathology at a tertiary care hospital. It was a hospital based cross - sectional study, done on outdoor and admitted patients during a 1 year period. All patients who presented with upper gastrointestinal [GI] symptoms such as dysphagia, vomiting, retrosternal pain, anorexia, loss of weight and mass abdomen were subjected to endoscopy. Endoscopy was done by using flexible video endoscope. Patients with visible red and white mucosal lesions, ulcers, polypoidal or ulcerative growth in upper GIT were included in the study. After visual examination of the lesion, a cytological brush made up of 
small nylon bristles at the tip was introduced through a separate channel in the endoscope .The brush was advanced up to the lesions so that the bristles touched the mucosa or suspected lesion, by up and down or rotary motions. The trapped exfoliated cells were smeared on the glass slides.

Brush smears were fixed in 95\% alcohol, and stained with haematoxylin and eosin (H\&E) and Papanicolaou stains. After brushing, biopsies were taken from suspicious area. The histopathological specimens were processed routinely and stained with haematoxylin and eosin (H\&E). The cytological and histopathological examination was carried out in the Department of Pathology. Smears were interpreted as inflammatory, fungal infection, dysplastic, suspicious of malignancy and positive for malignancy.

\section{Results}

In this study a total of 100 patients presented with upper GIT symptoms and detectable lesions on endoscopy. There were $60(60 \%)$ males and $40(40 \%)$ females. The male: female ratio was 3:2. The age of patients ranged from $20-80$ years. On endoscopy, 94 cases (94\%) showed esophageal lesions, 5 cases (5\%) showed gastric lesions and one case $(1 \%)$ showed duodenal lesion. The majority of the patients presented with common symptoms such as dysphagia, retrosternal burning and loss of weight. Dysphagia and retrosternal burning pain was the commonest symptoms found in all patients. The material obtained was adequate for cytology in all 100 cases.The positive rate of brush cytology with concomitant biopsy was $83.78 \%$. The overall results of brush cytology and biopsy examination in 100 patients with upper GIT lesions are shown in tables 1, 2 and 3 and in Figure 1.

\section{TABLES}

Table 1. Table showing the sites and sex distribution of the upper GIT lesions.

\begin{tabular}{|l|l|l|l|}
\hline Site of lesion & $\begin{array}{l}\text { Total number of brush } \\
\text { cytology } \\
\mathrm{n}=100\end{array}$ & Males & Females \\
\hline Oesophagus & 94 & $\mathrm{n}=60$ & $\mathrm{n}$ \\
\hline Gastric & 05 & 56 & 38 \\
\hline Duodenum & 01 & 03 & 02 \\
\hline
\end{tabular}

Table 2. Table showing cytology and histopathology availability with correlation.

\begin{tabular}{|l|l|l|}
\hline Procedure done & Total number of patients & Percentage \\
\hline Brush cytology done & 100 & \\
\hline Adequate cytology material & 100 & $100 \%$ \\
\hline Biopsy done & $67 / 100$ & $67 \%$ \\
\hline $\begin{array}{l}\text { Histopathology correlation } \\
\text { confirmed }\end{array}$ & $61 / 67$ & $83.78 \%$ \\
\hline Inadequate biopsy material & $06 / 67$ & $16.22 \%$ \\
\hline
\end{tabular}

Table 3. Table showing the distribution of the various types of lesions.

\begin{tabular}{|l|c|l|c|}
\hline Test results & $\begin{array}{l}\text { Total number of } \\
\text { patients } \\
\mathrm{n}=100\end{array}$ & $\begin{array}{l}\text { Adequate cytology } \\
\text { material } \\
\mathrm{n}=100\end{array}$ & $\begin{array}{l}\text { Adequate biopsy } \\
\text { material } \\
\mathrm{n}=61\end{array}$ \\
\hline Inflammatory & 20 & 20 & 2 \\
\hline Fungal infection & 13 & 13 & 3 \\
\hline Dysplastic & 42 & 42 & 36 \\
\hline $\begin{array}{l}\text { Suspicious for } \\
\text { malignancy }\end{array}$ & 4 & 4 & 2 \\
\hline Positive for malignancy & 21 & 21 & 18 \\
\hline
\end{tabular}




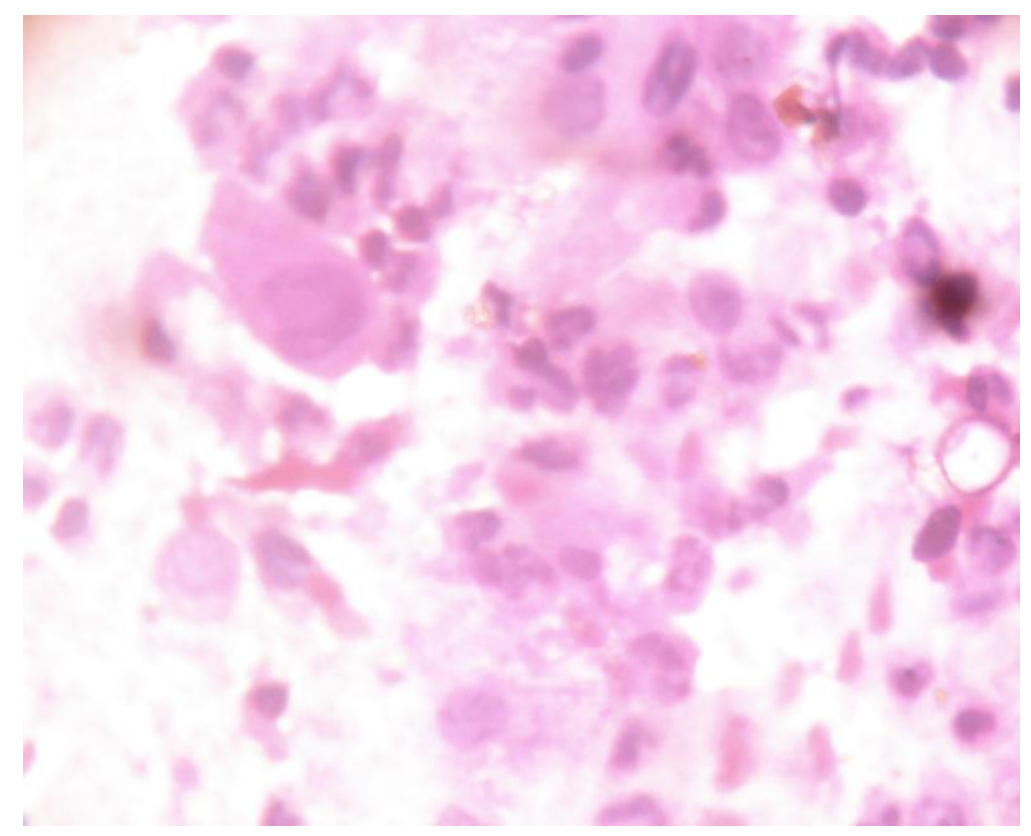

Figure 1. Cytology of oesophageal brushing showing a group of malignant cells. [Haematoxylin and Eosin X 400].

\section{Discussion}

Early gastrointestinal malignancies are asymptomatic and highly curable, but unfortunately most of them are symptomatic and advanced by the the time they are diagnosed. Early detection of a premalignant lesion can improve the survival and the morbidity. It can also improve the patient's quality of life, because less aggressive treatment is necessary. ${ }^{[6]}$ The introduction of flexible fiberoptic endoscope which allows brushing under direct visual control has greatly expanded the usefulness and precision of gastrointestinal tract cytology.

${ }^{[1,6]}$ Direct vision brush cytology of the upper GIT mucosa as obtained through the channel of the flexible endoscope is now a standard method of diagnosis either as an adjunct to tissue biopsy or as an independent method. The Japanese pioneered fiberoptic gastroscopy and brushing cytology because of the high rates of gastric carcinoma in Japan. In China, cytology is being used for the diagnosis of esophageal lesions. Thus diagnostic brushing cytology is a well established technique for the diagnosis of precancerous and cancerous lesions of upper gastrointestinal tract. In India very few institutions have adopted these techniques. ${ }^{[7]}$ Its reliability has been reported to range from $75 \%$ to $90 \%$ in various series. ${ }^{[8,9]}$

Cytology as a sole modality of diagnosis of the upper oesophageal lesions, especially in early detection of carcinoma, has been used in mass screening projects with good results in parts of the world where this disease is common. Most of the time a single biopsy, even if technically satisfactory, is insufficient to avoid the possibility of false negative report. ${ }^{[10,11,12]}$ Diagnostic yield of histopathology depends on several factors, such as the size, location and the level of experience.

The primary role of GIT cytology is cancer detection. Its potential using gastric washings, has been escribed even before the advent of endoscopes. Endoscopy not only allows the visualization of mucosal lesions, but at the same time, it permits the sampling of cytology and biopsy for a definitive diagnosis. Esophagus and gastric cancers are frequent cancers found in Indian men, while oesophageal cancer ranks third among women after carcinoma of the breast and cervix. Dietary factors are thought to be the most important etiological factor in squamous cell carcinoma. N- nitrosamines present in the food in high risk regions in China and Iran are found to be carcinogenic to esophagus. ${ }^{[13]}$ Early detection of malignancy greatly improves the survival rate of the patients. ${ }^{[6,10,14]}$ Early cancers are asymptomatic, and may be difficult for early diagnosis. Cytological method is well accepted by the patient and is an attractive option for the early diagnosis of upper GIT lesions. It is also useful when the lesion is large or multiple or the patients refuse biopsy. ${ }^{[10,11]}$ During our one year study period, 100 cases were included in which brush cytology was performed. Adequate cytology material was obtained in all the cases. Biopsy was done in 67 cases of which 6 cases diagnosed as dysplastic lesion on cytology showed inadequate biopsy material. This shows that the sensitivity of brushing is much higher than that of endoscopic 
biopsy. The brush cytology findings in the present study showed dysplastic lesions in $42 \%$, malignancy mostly squamous cell carcinoma in $21 \%$ and inflammation in $20 \%$ cases. Candida was seen in $13 \%$ cases. [Tables 1 , 2 and 3]. .Most of the lesions were from middle and lower part of oesophagus, cardiac end of stomach and first part of duodenum. The overall positivity of brush cytology in this study was $100 \%$.

In a study of 75 patients who presented with upper GIT lesions by K. Vidyavathi et al, ${ }^{[1]}$ the sensitivity of brush cytology was $98.03 \%$ and specificity was $81.11 \%$, which emphasizes the usefulness of brush cytology as a screening procedure. A study done by Wang et al ${ }^{[14]}$ indicated that combining cytology with biopsy increases the false positive rates, however, it also increases sensitivity of the procedure. However, a study done by Donoghue et al ${ }^{[12]}$ found that with the additional use of cytology, the sensitivity increased from $88.3 \%$ to $97.5 \%$. Therefore, it suggests that cytology is a useful adjunct in patients with suspicious mucosal lesions. ${ }^{[1,12,14]}$

Cytological brushing also has the many advantages such as acceptance by the patients, good penetration to the basement membrane, collecting cells from all three epithelial layers and early diagnosis of upper GIT lesions. ${ }^{[10,12]}$ There are many factors which influence the diagnostic accuracy of brush cytology. In order to improve the positive rate of brush cytology examination, some technical considerations should be noted such as the site for the specimen should be appropriately selected, avoiding lesions covered by thick slough or necrotic material and the procedure should be performed by a technically trained person. It should be performed at the margin of the ulcer and entire head of the nylon brush should be used over a wide area with appropriate force. After brushing is completed, the head of the brush should be withdrawn to the tip to prevent the loss of the specimen. During smearing of the slides, filter paper should be used to absorb excess mucus or clot adherent to the head of the brush. ${ }^{[5,15]}$

Biopsy and brush cytology may also provide false positive results. Smithies et al ${ }^{[16]}$ showed that the false negative rate of biopsy is higher than that of brush cytology, and false positive rate of cytology is higher than that of biopsy. Winawer et al ${ }^{[17]}$ found that the false positive rate is not high in the hands of an experienced examiner. Although multiple biopsies also increase the areas sampled, cytological brushing smears have the advantage of covering a relatively large area and tendency to selectively collect loose cells. This may explain the superiority of cytology in detecting malignancy. ${ }^{[1,5,16,17]}$

The limitation of cytology is its inability to distinguish between dysplasia, carcinoma in situ and invasive carcinoma. Another issue is that whether the brushing should be performed before or after biopsies. Some of the authors prefer to perform the brushing after biopsy believing that it might decrease the yield of biopsy. However, studies have shown that the accuracy of brushing cytology in patients with carcinoma was significantly higher when the brushing was performed before biopsy than after biopsy.

\section{Conclusion}

Although biopsy is used as a routine procedure in diagnosis of gastrointestinal tract lesions, brush cytology is useful because it is inexpensive, gives rapid diagnosis and offers minimal discomfort to the patient. A larger surface area can be sampled by brush cytology and it has an excellent specificity and good sensitivity for the detection of high grade dysplasia and in the identification of malignancy. By setting up such facilities in gastroenterology units, the patients are benefitted, as happened in our institute when we convinced the clinicians to take up the procedure. Although definitive surgical treatment is rarely based on a positive or suspicious cytological smear, it can alert the clinician about the possibility of malignancy. It is an accurate diagnostic adjunct that can be of significant value in early cancer detection. It is also useful as a screening procedure for a high risk population or for clinical follow up.

\section{References}

[1] Vidyavathi K, Harendrakumar ML, Lakshmana Kumar YC. Correlation of endoscopic brush cytology with biopsy in diagnosis of upper gastrointestinal neoplasms. Indian J Pathol Microbiol 2008; 51:489 -92.

[2] Zhang XF, Huang CM, Lu HS et al. Surgical treatment and prognosis of gastric cancer in 2613 patients. World J Gastroenterol 2004; 10: $3405-08$.

[3] Enzinger PC, Mayer RJ. Esophageal cancer. N Engl J Med 2003; 349: 2241 - 52.

[4] Jhala N, Jhala D. Gastrointestinal tract cytology. In: Atkinson BF editor. Atlas of Diagnostic Cytopathology. 2nd ed. Philadelphia: Saunders; 2004.

[5] Guangbi Y et al. Value of biopsy and brush cytology in the diagnosis of gastric cancer. Gut 1982; 23:774 -76.

[6] Divani S, Gallias S. Advantages and difficulties of brush cytology in the identification of early oral cancer. www. onk.ns.ac.rs/ Archive 2009; 17: 11-12.

[7] Yang H, Berner K, Mei Q, et al. Cytologic screeining for esophageal cancer in a high- risk population in Anyang County, China. Acta Cytol 2002; 46: 446 - 52 .

[8] Dowlatshahi K, Skinner DB, DeMecster TR, Zachary L, Bibbo M, Weid GL.Evaluation of brush cytology as an independent technique for detection of esophageal carcinoma. The Journal of Thoracic and Cardiovascular Surgery 1985; 89: 848 - 51.

[9] National Cancer Registry Programme. First All India Report 2001 -2002. Vol 1 Indian Council of Medical Research 2004, Bangalore, India.

[10] Young JA, Hughes HE, Lee FD. Evaluation of endoscopic brush and biopsy touch smear cytology and biopsy histology in the diagnosis of carcinoma of the lower oesophagus and cardia. J Clin Pathol 1980; 33: 811 - 14. 
[11] Navone R, Burlo P, Pich A, Pentenero M, Broccoleti R, MarsicoA et al. The impact of liquid-based oral cytology on the diagnosis of oral squamous dysplasia and carcinoma. Cytopathology 2007; 18:356- 60.

[12] O Donoghue JM, Horgan PG, ODonohoe MK et al. Adjunctive endoscopic brush cytology in the detection of upper gastrointestinal malignancy. Acta Cytol 1995; 39: $28-34$.

[13] Ashok L, Anand L, Jayanthi V. Epidemiology of cancer of the esophagus global and regional perspective. Gastroenterology Today 2005; 9: $75-9$.

[14] Wang HH, Jonasson JG, Ducatman BS. Brushing cytology of the upper gastrointestinal tract: Obsolete or not? Acta Cytol 1991; 35 : $195-98$.

[15] Kill J, Anderson D, Jensen OM. Biopsy and brush cytology in the diagnosis of gastric cancer. Scand J Gastroenterol 1979; 14: 18991

[16] Smithies A, Lavell D, Hishon S et al. Value of brush cytology in diagnosis of gastric cancer. Br Med. J 1975; 4: 326 - 8.

[17] Winawer S, Posner G, Lightdale CJ et al. Endoscopic diagnosis of advanced gastric cancer - factors influencing yield. Gastroenterology 1975; 69:1183-7. 